International Journal of Pure and Applied Mathematics

Volume 94 No. 5 2014, 649-660

ISSN: 1311-8080 (printed version); ISSN: 1314-3395 (on-line version)

url: http://www.ijpam.eu

doi: http://dx.doi.org/10.12732/ijpam.v94i5.2

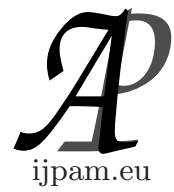

\title{
DECENTRALIZED EXPONENTIAL STABILIZATION OF LARGE-SCALE SYSTEMS WITH TIME-VARYING DELAY
}

\author{
Grienggrai Rajchakit \\ Department of Mathematics \\ Maejo University \\ Chiangmai, 50290, THAILAND
}

\begin{abstract}
This paper addresses decentralized exponential stabilization problem for a class of linear large-scale systems with time-varying delay in interconnection is considered. The time delay is any continuous function belonging to a given interval, but not necessary to be differentiable. By constructing a suitable augmented Lyapunov-Krasovskii functional combined with LeibnizNewton's formula, new delay-dependent sufficient conditions for the existence of decentralized exponential stability is established in terms of LMIs.
\end{abstract}

AMS Subject Classification: 93C30, 93D20, 37C75

Key Words: decentralized exponential stabilization, large-scale systems, interval time-varying delay, Lyapunov function, linear matrix inequalities

\section{Introduction}

Decomposition methods in the stability analysis of large-scale systems are becoming increasingly popular in the recent times and criteria for both asymptotic stability (in a Lyapunov setting) and input-output stability (in a functional analysis framework) have been obtained by treating the original system as an interconnection of several simpler subsystems. The problem of decentralized control of large-scale interconnected dynamical systems has been receiving considerable attention, because there are a large number of large-scale interconnected dynamical systems in many practical control problems, e.g., transportation systems, power systems, communication systems, economic systems, social

Received: January 12, 2014

(c) 2014 Academic Publications, Ltd. url: www.acadpubl.eu 
systems, and so on. [1-9]. The operation of large-scale interconnected systems requires the ability to monitor and stabilize in the face of uncertainties, disturbances, failures and attacks through the utilization of internal system states. However, even with the assumption that all the state variables are available for feedback control, the task of effective controlling a large-scale interconnected system using a global (centralized) state feedback controller is still not easy as there is a necessary requirement for information transfer between the subsystems [6-23]. To the best of our knowledge, there has been no investigation on the exponential stabilization of large-scale systems with time-varying delays interacted between subsystems. In fact, this problem is difficult to solve; particularly, when the time-varying delays are interval, non-differentiable and the output is subjected to such time-varying delay functions.

In this paper, we consider a class of large-scale linear systems with interval time-varying delays in interconnections. Compared to the existing results, our result has its own advantages. (i) stabilization analysis of previous papers reveals some restrictions: The time delay was proposed to be either time-invariant interconnected or the lower delay bound is restricted to being zero, or the time delay function should be differential and its derivative is bounded. In our result, the above restricted conditions are removed for the large-scale systems. In addition the time delay is assumed to be any continuous function belonging to a given interval, which means that the lower and upper bounds for the time-varying delay are available, but the delay function is bounded but not necessary to be differentiable. This allows the time-delay to be a fast time-varying function and the lower bound is not restricted to being zero. (ii) The developed method using new inequalities for lower bounding cross terms eliminate the need for over bounding and provide larger values of the admissible delay bound. We propose a set of new Lyapunov-Krasovskii functional, which are mainly based on the information of the lower and upper delay bounds.

The paper is organized as follows. Section 2 presents definitions and some well-known technical propositions needed for the proof of the main results. Main result for decentralized exponential stabilization of large-scale systems is presented in Section 3.

\section{Preliminaries}

The following noted in this paper. $R^{+}$denotes the set of all real non-negative numbers; $R^{n}$ denotes the $n$-dimensional space with the scalar product $\langle.,$. and the vector norm $\|.\| ; M^{n \times r}$ denotes the space of all matrices of $(n \times$ 
$r$ )-dimensions; $A^{T}$ denotes the transpose of matrix $A ; A$ is symmetric if $A=$ $A^{T} ; I$ denotes the identity matrix; $\lambda(A)$ denotes the set of all eigenvalues of $A ; \lambda_{\min / \max }(A)=\min / \max \{\operatorname{Re} \lambda ; \lambda \in \lambda(A)\} ; C^{1}\left([a, b], R^{n}\right)$ denotes the set of all $R^{n}$-valued differentiable functions on $[a, b] ; L_{2}\left([0, \infty], R^{r}\right)$ stands for the set of all square-integrable $R^{r}$-valued functions on $[0, \infty] . \quad x_{t}:=\{x(t+s)$ : $s \in[-h, 0]\},\left\|x_{t}\right\|=\sup _{s \in[-h, 0]}\|x(t+s)\| ; C\left([0, t], R^{n}\right)$ denotes the set of all $R^{n}$-valued continuous functions on $[0, t]$; Matrix $A$ is called semi-positive definite $(A \geq 0)$ if $\langle A x, x\rangle \geq 0$, for all $x \in R^{n} ; A$ is positive definite $(A>0)$ if $\langle A x, x\rangle>0$ for all $x \neq 0 ; A>B$ means $A-B>0$. * denotes the symmetric term in a matrix.

Consider a class of linear large-scale systems with interval time-varying delays composed of $N$ interconnected subsystems $i=\overline{1, N}$ of the form

$$
\begin{gathered}
\dot{x}_{i}(t)=A_{i} x_{i}(t)+\sum_{j \neq i, j=1}^{N} D_{i j} u_{j}(t), \quad t \in R^{+}, \\
x_{i}(t)=\varphi_{i}(t), \quad \forall t \in\left[-h_{2}, 0\right],
\end{gathered}
$$

where $x^{T}(t)=\left[x_{1}^{T}(t), \ldots, x_{N}^{T}(t)\right], x_{i}(t) \in R^{n_{\mathrm{i}}}$, is the state vector,$u_{j}(t) \in$ $R^{m_{j}}, m_{j} \leq n_{j}, j=\overline{1, N}$ is the control input, the systems matrices $A_{i}, D_{i j}$ are of appropriate dimensions.

We consider a delayed feedback control law

$$
u_{j}(t)=F_{i j} x_{j}\left(t-h_{i j}(t)\right), \quad t \in R^{+},
$$

and $F_{i j}, i, j=\overline{1, N}$ is the controller gain to be determined.

Remark 1. Note that to implement the delayed state feedback controller (1) the function $h_{i j}(t)$ must be known in advance. Applying the feedback controller (2) to the system (1), the closed-loop linear large-scale systems is

$$
\dot{x}_{i}(t)=A_{i} x_{i}(t)+\sum_{j \neq i, j=1}^{N} D_{i j} F_{i j} x_{j}\left(t-h_{i j}(t)\right), \quad t \in R^{+},
$$

The time delays $h_{i j}($.$) are continuous and satisfy the following condition:$

$$
0 \leq h_{1} \leq h_{i j}(t)<h_{2}, \quad t \geq 0, \quad \forall i, j=\overline{1, N},
$$

and the initial function $\varphi(t)=\left[\varphi_{1}(t), \ldots \varphi_{N}(t)^{T}\right], \varphi_{i}(t) \in C^{1}\left(\left[-h_{2}, 0\right], R^{n_{\mathrm{i}}}\right)$, with the norm

$$
\left\|\varphi_{i}\right\|=\sup _{-h \leq t \leq 0}\left\{\left\|\varphi_{i}(t)\right\|,\left\|\dot{\varphi}_{i}(t)\right\|\right\}, \quad\|\varphi\|=\sqrt{\sum_{i=1}^{N}\left\|\varphi_{i}\right\|^{2}}
$$


Definition 1. Given $\alpha>0$. The zero solution of system (1) is $\alpha$-exponentially stable if there exist a positive number $N>0$ such that every solution $x(t, \varphi)$ satisfies the following condition:

$$
\|x(t, \varphi)\| \leq N e^{-\alpha t}\|\varphi\|, \quad \forall t \in R^{+}
$$

Definition 2. The linear large-scale control systems (1) is exponentially stablilizable if there is a delayed feedback control (2) such that the linear largescale control systems (3) is exponentially stable.

We end this section with the following technical well-known propositions, which will be used in the proof of the main results.

Proposition 1. For any $x, y \in R^{n}$ and positive definite matrix $P \in R^{n \times n}$, we have

$$
2 x^{T} y \leq y^{T} P y+x^{T} P^{-1} x .
$$

Proposition 2. (Schur complement lemma [24]). Given constant matrices $X, Y, Z$ with appropriate dimensions satisfying $X=X^{T}, Y=Y^{T}>0$. Then $X+Z^{T} Y^{-1} Z<0$ if and only if

$$
\left(\begin{array}{cc}
X & Z^{T} \\
Z & -Y
\end{array}\right)<0 \quad \text { or } \quad\left(\begin{array}{cc}
-Y & Z \\
Z^{T} & X
\end{array}\right)<0
$$

Proposition 3. [25] For any constant matrix $Z=Z^{T}>0$ and scalar $h, \bar{h}, 0<h<\bar{h}$ such that the following integrations are well defined, then

$$
\begin{gathered}
-\int_{t-h}^{t} x(s)^{T} Z x(s) d s \leq-\frac{1}{h}\left(\int_{t-h}^{t} x(s) d s\right)^{T} Z\left(\int_{t-h}^{t} x(s) d s\right) \\
-\int_{-\bar{h}}^{-h} \int_{t+\theta}^{t} x(s)^{T} Z x(s) d s d \theta \leq-\frac{2}{\bar{h}^{2}-h^{2}}\left(\int_{-\bar{h}}^{-h} \int_{t+\theta}^{t} x(s) d s d \theta\right)^{T} \\
Z\left(\int_{-\bar{h}}^{-h} \int_{t+\theta}^{t} x(s) d s d \theta\right) .
\end{gathered}
$$

\section{Main Results}

In this section, we investigate the decentralized exponential stabilization of linear large-scale control system (1) with interval time-varying delays. It will be seen from the following theorem that neither free-weighting matrices nor any 
transformation are employed in our derivation. Before introducing main result, the following notations of several matrix variables are defined for simplicity.

$$
\begin{aligned}
& M_{11}^{i}= A_{i}^{T} P_{i}+A_{i} P_{i}+2 \alpha P_{i}-\left(\frac{2 e^{-4 \beta h_{2}}}{h_{2}^{2}-h_{1}^{2}}\right)\left(h_{2}-h_{1}\right)^{2} W_{i} \\
&+\sum_{j \neq i, j=1}^{N} P_{i}\left(D_{i j} F_{i j}\right)\left(D_{i j} F_{i j}\right)^{T} P_{i}+S_{i 3} A_{i} A_{i}^{T} S_{i 3}^{T}, \\
& M_{1 k}^{i}=-S_{i 3} A_{i}, \forall k=\overline{2, N}, M_{1(N+1)}^{i}=-S_{i 1} A_{i}, \\
& M_{1(N+2)}^{i}= S_{i 2} A_{i}, M_{1(N+3)}^{i}=0, M_{1(N+4)}^{i}=\left(\frac{2 e^{-4 \beta h_{2}}}{h_{2}^{2}-h_{1}^{2}}\right)\left(h_{2}-h_{1}\right) W_{i}, \\
& M_{k m}^{i}=0, \forall k \neq m, k, m=\overline{2, N}, \\
& M_{k k}^{i}=\sum_{j \neq i, j=1}^{N} S_{i 3}\left(D_{i j} F_{i j}\right)\left(D_{i j} F_{i j}\right)^{T} S_{i 4}^{T}+6 I, \forall k=\overline{2, N}, \\
& M_{k(N+1)}^{i}=0, M_{k(N+2)}^{i}=0, M_{k(N+3)}^{i}=S_{i 3}, M_{k(N+4)}^{i}=0, \\
& M_{(N+1)(N+1)}^{i}=\sum_{j \neq i, j=1}^{N} S_{i 1}\left(D_{i j} F_{i j}\right)\left(D_{i j} F_{i j}\right)^{T} S_{i 1}^{T}, M_{(N+1)(N+2)}^{i}=0, \\
& M_{(N+1)(N+3)}^{i}=S_{i 1}, M_{(N+1)(N+4)}^{i}=0, \\
& M_{(N+2)(N+2)}^{i}=\sum_{j \neq i, j=1}^{N} S_{i 2}\left(D_{i j} F_{i j}\right)\left(D_{i j} F_{i j}\right)^{T} S_{i 2}^{T}, M_{(N+2)(N+3)}^{i}=S_{i 2}, \\
& M_{(N+2)(N+4)}^{i}=S_{i 1}, M_{(N+3)(N+3)}^{i}=2 S_{i 4}+\left(h_{2}-h_{1}\right) h_{2} W_{i} \\
&+\sum_{j \neq i, j=1}^{N} S_{i 4}\left(D_{i j} F_{i j}\right)\left(D_{i j} F_{i j}\right)^{T} S_{i 4}^{T}, \\
& \lambda_{(N+3)(N+4)}=0, M_{(N+4)(N+4)}^{i}=\left(\frac{2 e^{-4 \beta h_{2}}}{h_{2}^{2}-h_{1}^{2}}\right) W_{i}, \\
& M_{m a x}\left(P_{i}\right), \lambda_{1}=m_{i=1, N} \lambda_{i 1}, \lambda_{2}=m_{i=\overline{1, N}} \lambda_{i 2}, \\
&\left.+h_{2}-h_{1}\right) h_{2}^{2} \lambda_{m a x}\left(W_{i}\right) .
\end{aligned}
$$

The following is the main result of the paper, which gives sufficient conditions for the decentralized exponential stabilization of linear large-scale system (1) with interval time-varying delays. Essentially, the proof is based on the construction of Lyapunov Krasovskii functions satisfying Lyapunov stability theorem for time-delay system [24]. 
Theorem 1. Given $\alpha>0$. System (1) is $\alpha$-exponentially stable if there exist symmetric positive definite matrices $P_{i}, W_{i}, i=\overline{1, N}$, and matrices $S_{i j}, i=$ $\overline{1, N}, j=1,2,3,4$ such that the following LMI holds

$$
\mathcal{M}^{i}=\left[\begin{array}{cccc}
M_{11}^{i} & M_{12}^{i} & \ldots & M_{1(N+4)}^{i} \\
* & M_{22}^{i} & \ldots & M_{2(N+4)}^{i} \\
\cdot & \cdot & \ldots & \cdot \\
* & * & \ldots & M_{(N+4)(N+4)}^{i} \cdot
\end{array}\right]<0, \quad i=\overline{1, N}
$$

Moreover, the solution $x(t, \varphi)$ of the system satisfies

$$
\|x(t, \varphi)\| \leq \sqrt{\frac{\lambda_{2}}{\lambda_{1}}} e^{-\alpha t}\|\varphi\|, \quad \forall t \in R^{+} .
$$

Proof. We consider the following Lyapunov-Krasovskii functional for the system (1)

$$
V\left(t, x_{t}\right)=\sum_{i=1}^{N} \sum_{j=1}^{2} V_{i j}\left(t, x_{t}\right)
$$

where

$$
\begin{aligned}
V_{i 1} & =x_{i}^{T}(t) P_{i} x_{i}(t) \\
V_{i 2} & =\int_{-h_{2}}^{-h_{1}} \int_{\theta}^{0} \int_{t+s}^{t} e^{2 \alpha(\tau+s-t)} \dot{x}_{i}^{T}(\tau) W_{i} \dot{x}_{i}(\tau) d \tau d s d \theta
\end{aligned}
$$

It easy to verify that

$$
\sum_{i=1}^{N} \lambda_{i 1}\left\|x_{i}(t)\right\|^{2} \leq V\left(t, x_{t}\right), \quad V\left(0, x_{0}\right) \leq \sum_{i=1}^{N} \lambda_{i 2}\left\|\varphi_{i}\right\|^{2} .
$$

Taking the derivative of $V$ in $t$ along the solution of system (1), we have

$$
\begin{aligned}
& \dot{V}_{i 1}=2 x_{i}^{T}(t) P_{i} \dot{x}_{i}(t) \\
& =2 x_{i}^{T}(t)\left[A_{i}^{T} P_{i}+A_{i} P_{i}\right] x_{i}(t)+2 x_{i}^{T}(t) P_{i} D_{i j} F_{i j} x_{j}\left(t-h_{i j}(t)\right) ; \\
& \dot{V}_{i 2} \leq\left(h_{2}-h_{1}\right) h_{2} \dot{x}_{i}^{T}(t) W_{i} \dot{x}_{i}(t) \\
& \quad-e^{-4 \beta h_{2}} \int_{-h_{2}}^{-h_{1}} \int_{t+\theta}^{t} \dot{x}_{i}^{T}(s) W_{i} \dot{x}_{i}(s) d s d \theta-2 \alpha V_{i 2} .
\end{aligned}
$$


Note that when $h_{j i}(t)=h_{1}$ or $h_{j i}(t)=h_{2}$, we have

$$
\left[x_{i}\left(t-h_{1}\right)-x_{i}\left(t-h_{j i}(t)\right)\right]^{T}=0
$$

or

$$
\left[x_{i}\left(t-h_{j i}(t)\right)-x_{i}\left(t-h_{2}\right)\right]=0,
$$

respectively. Besides, using Proposition 3 again, we have

$$
\begin{aligned}
& e^{-4 \beta h_{2}} \int_{-h_{2}}^{-h_{1}} \int_{t+\theta}^{t} \dot{x}_{i}^{T}(s) W_{i} \dot{x}_{i}(s) d s d \theta \leq \\
& -e^{-4 \beta h_{2}} \frac{2}{\bar{h}^{2}-h^{2}}\left(\int_{-\bar{h}}^{-h} \int_{t+\theta}^{t} x(s) d s d \theta\right)^{T} Z\left(\int_{-\bar{h}}^{-h} \int_{t+\theta}^{t} x(s) d s d \theta\right) \leq \\
& \frac{-2 e^{-4 \beta h_{2}}}{h_{2}^{2}-h_{1}^{2}}\left(\left(h_{2}-h_{1}\right) x_{i}(t)-\int_{t-h_{2}}^{t-h_{1}} x_{i}(\theta) d \theta\right)^{T} \\
& W_{i}\left(\left(h_{2}-h_{1}\right) x_{i}(t)-\int_{t-h_{2}}^{t-h_{1}} x_{i}(\theta) d \theta\right) .
\end{aligned}
$$

Hence,

$$
\begin{aligned}
\dot{V}_{i 2} \leq & \left(h_{2}-h_{1}\right) h_{2} \dot{x}_{i}^{T}(t) W_{i} \dot{x}_{i}(t)-2 \alpha V_{i 2} \\
& -\frac{2 e^{-4 \beta h_{2}}}{h_{2}^{2}-h_{1}^{2}}\left(\left(h_{2}-h_{1}\right) x_{i}(t)-\int_{t-h_{2}}^{t-h_{1}} x_{i}(\theta) d \theta\right)^{T} \\
& \times W_{i}\left(\left(h_{2}-h_{1}\right) x_{i}(t)-\int_{t-h_{2}}^{t-h_{1}} x_{i}(\theta) d \theta\right) .
\end{aligned}
$$

Therefore, we have

$$
\begin{aligned}
\dot{V}(.)+2 \alpha V(.) \leq & \sum_{i=1}^{N}\left[x _ { i } ^ { T } ( t ) \left[A_{i}^{T} P_{i}+A_{i} P_{i}+2 \alpha P_{i}-\left(\frac{2 e^{-4 \beta h_{2}}}{h_{2}^{2}-h_{1}^{2}}\right)\left(h_{2}-h_{1}\right)^{2} W_{i}\right.\right. \\
& \left.+\sum_{j \neq i, j=1}^{N} P_{i}\left(D_{i j} F_{i j}\right)\left(D_{i j} F_{i j}\right)^{T} P_{i}+S_{i 3} A_{i} A_{i}^{T} S_{i 3}^{T}\right] x_{i}(t) \\
& \left.+\sum_{j \neq i, j=1}^{N} x_{i}^{T}\left(t-h_{1}\right) S_{i 1}\left(D_{i j} F_{i j}\right)\left(D_{i j} F_{i j}\right)^{T} S_{i 1}^{T}\right] x_{i}\left(t-h_{1}\right) \\
& +x_{i}^{T}\left(t-h_{2}\right)\left[\sum_{j \neq i, j=1}^{N} S_{i 2}\left(D_{i j} F_{i j}\right)\left(D_{i j} F_{i j}\right)^{T} S_{i 2}^{T}\right] x_{i}\left(t-h_{2}\right)
\end{aligned}
$$




$$
\begin{aligned}
& +\dot{x}_{i}^{T}(t)\left[2 S_{i 4}+\left(h_{2}-h_{1}\right) h_{2} W_{i}\right. \\
& \left.+\sum_{j \neq i, j=1}^{N} S_{i 4}\left(D_{i j} F_{i j}\right)\left(D_{i j} F_{i j}\right)^{T} S_{i 4}^{T}\right] \dot{x}_{i}(t) \\
& +\sum_{j \neq i, j=1}^{N} x_{i}^{T}\left(t-h_{j i}(t)\right)\left[\sum_{j \neq i, j=1}^{N} S_{i 3}\left(D_{i j} F_{i j}\right)\left(D_{i j} F_{i j}\right)^{T} S_{i 3}^{T}\right. \\
& +6 I] x_{i}\left(t-h_{j i}(t)\right) \\
& \left.+2 x_{i}^{T}(t)\left[-S_{i 1} A_{i}\right] x_{i}\left(t-h_{1}\right)\right]+\sum_{i=1}^{N}\left[2 x_{i}^{T}(t)\left[S_{i 2} A_{i}\right] x_{i}\left(t-h_{2}\right)\right. \\
& +2 x_{i}^{T}(t)\left[-S_{i 4} A_{i}\right] \dot{x}_{i}(t)+2 x_{i}^{T}\left(t-h_{1}\right) S_{i 1} \dot{x}_{i}(t) \\
& +2 x_{i}^{T}\left(t-h_{1}\right) S_{i 2} \dot{x}_{i}(t)+2 \sum_{j \neq i, j=1}^{N} x_{i}^{T}\left(t-h_{j i}(t)\right) S_{i 3} \dot{x}_{i}(t) \\
& -\left(\int_{t-h_{2}}^{t-h_{1}} x_{i}(\theta) d \theta\right)^{T}\left[\left(\frac{2 e^{-4 \beta h_{2}}}{h_{2}^{2}-h_{1}^{2}}\right) W_{i}\right]\left(\int_{t-h_{2}}^{t-h_{1}} x_{i}(\theta) d \theta\right) \\
& +2 x_{i}^{T}(t)\left[\left(\frac{2 e^{-4 \beta h_{2}}}{h_{2}^{2}-h_{1}^{2}}\right)\left(h_{2}-h_{1}\right) W_{i}\right]\left(\int_{t-h_{2}}^{t-h_{1}} x_{i}(\theta) d \theta\right) \\
& =\sum_{i=1}^{N} \zeta_{i}^{T}(t) \mathcal{M}^{i} \zeta_{i}(t),
\end{aligned}
$$

where

$$
\begin{aligned}
& \zeta_{i}^{T}(t)= \\
& {\left[x_{i}^{T}(t), x_{i}^{T}\left(t-h_{1}\right), x_{i}^{T}\left(t-h_{2}\right),\left(x_{i}^{T}\left(t-h_{j i}\right)\right)_{j \neq i, j=1}^{N}, \dot{x}_{i}^{T}(t), \int_{t-h_{2}}^{t-h_{1}} x_{i}^{T}(\theta) d \theta\right] .}
\end{aligned}
$$

By condition (4), we obtain

$$
\dot{V}\left(t, x_{t}\right) \leq-2 \alpha V\left(t, x_{t}\right), \quad \forall t \in R^{+} .
$$

Integrating both sides of (6) from 0 to $t$, we obtain

$$
V\left(t, x_{t}\right) \leq V(\varphi) e^{-2 \alpha t}, \quad \forall t \in R^{+} .
$$

Furthermore, taking condition (5) into account, we have

$$
\lambda_{1}\|x(t, \varphi)\|^{2} \leq V\left(x_{t}\right) \leq V(\varphi) e^{-2 \alpha t} \leq \lambda_{2} e^{-2 \alpha t}\|\varphi\|^{2},
$$


then

$$
\|x(t, \varphi)\| \leq \sqrt{\frac{\lambda_{2}}{\lambda_{1}}} e^{-\alpha t}\|\varphi\|, \quad t \in R^{+} .
$$

This completes the proof of the theorem.

Remark 2. Theorem 1 provides sufficient conditions for linear large-scale system (1) in terms of the solutions of LMIs, which guarantees the closed-loop system to be exponentially stable with a prescribed decay rate $\alpha$. The developed method using new inequalities for lower bounding cross terms eliminate the need for over bounding and provide larger values of the admissible delay bound. Note that the time-varying delays are non-differentiable, therefore, the methods proposed in $[1-5,9-12,15,18,20-23]$ are not applicable to system (1). The LMI condition (4) depends on parameters of the system under consideration as well as the delay bounds.

\section{Conclusion}

In this paper, the problem of the decentralized exponential stabilization for large-scale time-varying delay systems has been studied. The time delay is assumed to be a function belonging to a given interval, but not necessary to be differentiable. The developed method using new inequalities for lower bounding cross terms eliminate the need for over-bounding and provide larger values of the delay bound.

\section{Acknowledgments}

This work was supported by the Thailand Research Fund Grant, the Commission for Higher Education and Faculty of Science, Maejo University, Thailand. The authors thank anonymous reviewers for valuable comments and suggestions, which allowed us to improve the paper.

\section{References}

[1] Kreangkri Ratchagit, STABILITY CRITERIA OF LPD SYSTEM WITH TIME-VARYING DELAY, International Journal of Pure and Applied Mathematics, Vol. 78, No. 6, 2012, 857-866. 
[2] Kreangkri Ratchagit, STABILITY ANALYSIS OF LINEAR SYSTEMS WITH TIME DELAYS, International Journal of Pure and Applied Mathematics, Vol. 76, No. 1, 2012, 21-28.

[3] Kreangkri Ratchagit , STABILITY OF LINEAR TIME-VARYING SYSTEMS, International Journal of Pure and Applied Mathematics, Vol. 63, No. 4, 2010, 411-417.

[4] Kreangkri Ratchagit , EXPONENTIAL STABILITY OF SWITCHED LINEAR SYSTEMS, International Journal of Pure and Applied Mathematics, Vol. 58, No. 3, 2010, 361-371.

[5] K. Ratchagit , THE SUFFICIENT CONDITIONS FOR STABILITY OF LINEAR TIME-VARYING SYSTEMS WITH STATE DELAYS, International Journal of Pure and Applied Mathematics, Vol. 65, No. 1, 2010, 65-72.

[6] K. Ratchagit and V.N. Phat, Stability criterion for discrete-time systems, Journal of Inequalities and Applications, 2010, 2010:201459 doi:10.1155/2010/201459.

[7] G. Rajchakit, Switching design for the robust stability of nonlinear uncertain stochastic switched discrete-time systems with interval time-varying delay. Journal of Computational Analysis and Applications, Vol. 16, 2014, 10-19.

[8] M. De la Sen and A. Ibeas, Stability Results of a Class of Hybrid Systems under Switched Continuous-Time and Discrete-Time Control, Discrete Dynamics in Nature and Society, Vol. 2009, Article ID 315713, 28 pages, 2009. doi:10.1155/2009/315713

[9] G. Rajchakit, Robust stability and stabilization of nonlinear uncertain stochastic switched discrete-time systems with interval time-varying delays, APPLIED MATHEMATICS and INFORMATION SCIENCES, Vol. 6, 2012, 555-565.

[10] Manlika Rajchakit, Piyapong Niamsup, Grienggrai Rajchakit, LMI approach to decentralized exponential stability of linear large-scale systems with interval non-differentiable time-varying delays, ADVANCES IN DIFFERENCE EQUATIONS, 2013. DOI: 10.1186/1687-1847-2013-332 
[11] G. Rajchakit, Delay-dependent optimal guaranteed cost control of stochastic neural networks with interval nondifferentiable time-varying delays, ADVANCES IN DIFFERENCE EQUATIONS, 2013, 1-11. DOI: 10.1186/1687-1847-2013-241

[12] K. Ratchagit, A switching rule for the asymptotic stability of discrete-time systems with convex polytopic uncertainties, Asian-European Journal of Mathematics, Vol. 5, 2012. DOI: 10.1142/S1793557112500258

[13] G. Rajchakit, Stabilization of switched discrete-time systems with convex polytopic uncertainties, Journal of Computational Analysis and Applications, Vol. 16, 2014, 20-29.

[14] Manlika Rajchakit, Piyapong Niamsup, Grienggrai Rajchakit, A constructive way to design a switching rule and switching regions to mean square exponential stability of switched stochastic systems with non-differentiable and interval time-varying delay, Journal of Inequalities and Applications, 2013, 2013:499. doi:10.1186/1029-242X-2013-499

[15] Grienggrai Rajchakit, Switching Design for the Asymptotic Stability and Stabilization of Nonlinear Uncertain Stochastic Discrete-time Systems, INTERNATIONAL JOURNAL OF NONLINEAR SCIENCES AND NUMERICAL SIMULATION, Vol. 14, 2013, 33-44. DOI: 10.1515/ijnsns2011-0176

[16] Grienggrai Rajchakit, Delay-Dependent Asymptotical Stabilization Criterion of Recurrent Neural Networks, Applied Mechanics and Materials. Vol. 330, 2013, 1045-1048. doi:10.4028/www.scientific.net/AMM.330.1045

[17] K. Ratchagit, Asymptotic stability of nonlinear delay-difference system via matrix inequalities and application, International Journal of Computational Methods, Vol. 6, 2009, 389-397. DOI: 10.1142/S0219876209001899

[18] M. de la Sen, Global Stability of Polytopic Linear Time-Varying Dynamic Systems under Time-Varying Point Delays and Impulsive Controls, Mathematical Problems in Engineering, vol. 2010, Article ID 693958, 33 pages, 2010. doi: $10.1155 / 2010 / 693958$

[19] G. Rajchakit, Exponential stability of switched linear systems with interval time-varying delays, Proceedings of the 2012 IEEE International Conference on Robotics and Biomimetics, December 11-14, 2012, Guangzhou, China, 1502-1506. doi: 10.1109/ROBIO.2012.6491181 
[20] K. Ratchagit, V.N. Phat, Stability and stabilization of switched linear discrete-time systems with interval time-varying delay, Nonlinear Anal. Hybrid Syst., Vol. 5, 2011, 605-612. DOI: 10.1016/j.nahs.2011.05.006

[21] Manlika Rajchakit, Piyapong Niamsup, Grienggrai Rajchakit, A constructive way to design a switching rule and switching regions to mean square exponential stability of switched stochastic systems with non-differentiable and interval time-varying delay, JOURNAL OF INEQUALITIES AND APPLICATIONS, 2013. DOI: 10.1186/1029-242X-2013-499

[22] VN. Phat, Y. Kongtham, and K. Ratchagit, LMI approach to exponential stability of linear systems with interval time-varying delays, Linear Algebra Appl., Vol. 436, 2012, 243-251. doi: 10.1016/j.laa.2011.07.016

[23] P. Niamsup, M. Rajchakit, G. Rajchakit, Guaranteed cost control for switched recurrent neural networks with interval time-varying delay, JOURNAL OF INEQUALITIES AND APPLICATIONS, 2013. DOI: 10.1186/1029-242X-2013-292

[24] P. Niamsup, G. Rajchakit, New Results on Robust Stability and Stabilization of Linear Discrete-Time Stochastic Systems with Convex Polytopic Uncertainties, JOURNAL OF APPLIED MATHEMATICS, 2013. DOI: $10.1155 / 2013 / 368259$

[25] R.P. Agarwal, Difference Equations and Inequalities, Second Edition, Marcel Dekker, New York, 2000. 